\title{
Photometric Linearization under Near Point Light Sources
}

\author{
Satoshi Sato, Kazutoyo Takata and Kunio Nobori \\ Advanced Technology Research Laboratories, Matsushita Electric Industrial Co., Ltd. \\ 3-4, Hikari-dai, Seika-cho, Soraku-gun, Kyoto, 619-0237, Japan \\ E-mail: \{sato.satoshi, nobori.kunio, takata.kazutoyo\}@jp.panasonic.com
}

\begin{abstract}
We present a method for generating linearized images that consist of only diffuse reflection from images taken under near point light sources. Previous photometric linearization method cannot work under near point light sources, since it assumes parallel light. For satisfying this assumption, our method utilizes a photometric linearization method by dividing images into small regions. It also selects linearization coefficients from regions. Experimental results show that the proposed method can generate accurate linearized images even if images are obtained under near point light sources, and show that the method can correctly classifies the photometric factors.
\end{abstract}

\section{Introduction}

The generation of images without specular reflection and shadows from real images is one of the common topics in computer graphics and object recognition. For this purpose, methods for classifying photometric factors such as specular reflection, diffuse reflection, attached shadows and cast shadows (Figure 1), have been proposed.

For example, Umeyama et al. [7] separate diffuse and specular components of surface reflection as two independent components by applying Independent Component Analysis to the images observed through a polarizer of different orientations. Swaminathan et al. [6] classify specular reflection regions on the basis of epipolar plane images (EPI) obtained from a moving camera. Ikeuchi et al. [2] classify diffuse reflection, specular reflection, and shadows using the subject's three-dimensional shape data obtained by a range finder.

Mukaigawa et al. [4], meanwhile, propose a photometric linearization (PL) method. This method can generate linearized images that consist of only diffuse reflection by applying the random sample consensus (RANSAC) algorithm [1] to real images containing shadows and specular reflection. And using the PL method, Ishii et al. [3], classify diffuse reflection, specular reflection, attached shadows, and cast shadows (Figure 1).

The PL method uses several intensity images obtained under different lighting condition. So the advantage of the PL method is that complex equipment such as polarizing filters and range finders is unnecessary.

The previous PL method, however, assume that the light source is parallel light, which means a point light source at infinity. In other words, this method can be used only in outdoor environments for which a parallel light source can be assumed. Therefore, it is difficult to apply PL method to general lighting environment.

We propose a new method for applying PL method [1] to more general lighting environment having near point

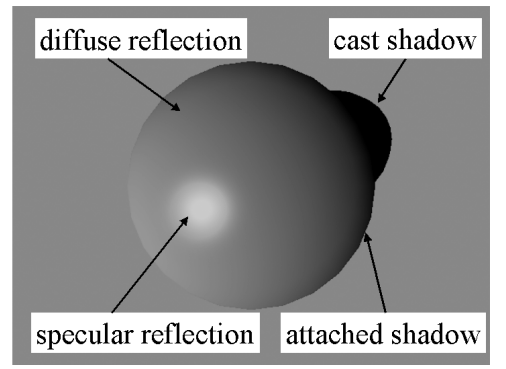

Figure 1: Various photometric factors

light sources. For achieving this goal, our method first divides images into small regions. Second, it selects the position and size of regions, and third, choices sets of linearization coefficients from the regions, to obtain accurate linearized images.

\section{Photometric Linearization under Near Point Light Sources}

\subsection{Issues in PL method by dividing images into}

\section{small regions}

The previous PL method requires the following preconditions.

Precondition 1: The light source is a point light source at infinity.

Precondition 2: Each image must include at least three diffuse pixels with different normal.

Precondition 3: Diffuse reflection is dominant in each image.

If the distance between the light sources and all points on objects in projected area in image is approximately constant, and if the distance is sufficiently longer than the area size, precondition 1 is satisfied. Therefore, we divide images into small regions and perform linearization processing for each region. A small region subjected to linearization processing is called a "process region." Although precondition 1 becomes satisfied as the process region becomes smaller, preconditions 2 and 3 will be violated. This is the reason why the smaller the size of process region is, the less the number of the diffuse pixels with different normal is.

To overcome above problem, we investigate the following two techniques.

Adaptive window (position and size): If the position and size of process region are not optimal, preconditions 2 and 3 might be violated. We therefore search an optimal process region from multiple sets of process region with different position and size.

Propagation of linearization coefficients: Even if we 
select the position and size of process region by the above technique, preconditions 2 and 3 might be violated. To overcome above problem, we select sets of linearization coefficients from neighboring regions.

\subsection{Adaptive window}

We search for optimal dividing of process regions with varying their positions and sizes.

Figure 2 shows an image of a spherical object placed on a plane. Broken lines in Figures 2(a) and (b) show how process regions can be positioned differently. We examine Point A contained by process regions both $\mathrm{R} 1$ and $\mathrm{R} 2$. To this case, region R1 includes only the plane and consequently fails to satisfy Precondition 2. Region R2, on the other hand, also includes part of the sphere, and therefore satisfies Precondition 2 and Precondition 3. As a result, a base image can be generated at region $\mathrm{R} 2$ but not at region R1.

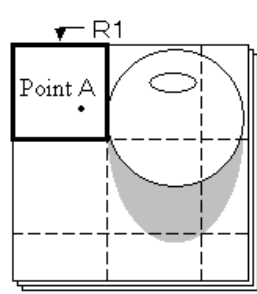

(a)

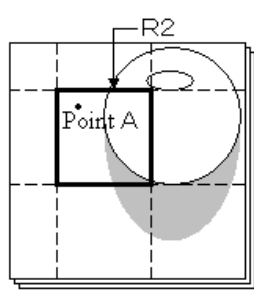

(b)

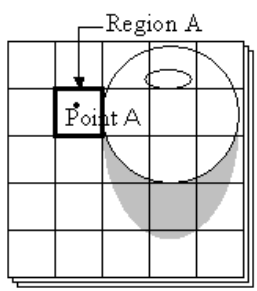

(c)
Figure 2: Selecting the position of process region

We determine multiple sets of base image candidates while changing the position and size of process region as in Figures 2(a) and (b). Then, as shown by solid lines in Figure 2(c), we further divide the process regions to obtain the base regions. For each of these base regions, we select an optimal base image from the candidates obtained above.

At this time, some kind of criterion is needed to determine which base image candidate is suitable for each base region. A typical approach is to measure the similarity between a base image candidate and the corresponding input image. However, input images for the PL method are obtained under different lighting condition. Therefore, to provide a criterion robust against changes in lighting, we adapt the similarity of Qualitative Trinary Representation (QTR) [3] based on the difference in intensities of adjacent pixels.

\subsection{Propagation of linearization coefficients}

The set of linearization coefficients of a process region has a correlation with those of neighboring, because they contain information on light source location. Accordingly, when a process region violates preconditions, it can be used the set of linearization coefficients of a neighboring region.

Note that in the event that no set of linearization coefficients was found for a process region, we select the linearization coefficients from eight neighboring regions. We then select the set that provides the most similar intensity to neighboring regions at the boundary pixels.

\subsection{Process flow}

We classify photometric factors with three steps, as shown in Figure 3.

\section{(1) Generating base images}

1) Divide all input images into process regions of dif- ferent positions and sizes.

2) Linearize each image at process region and generate one set of three base images on the region by PL method [1].

3) Select the most correct base image for each base region using QTR, and combine those base images to obtain base images $\mathbf{I}_{1}^{\mathrm{B}}, \mathbf{I}_{2}^{\mathrm{B}}$, and $\mathbf{I}_{3}^{\mathrm{B}}$.

(2) Linearization of input image

1) Divide input image $\mathbf{I}_{k}$ into process regions.

2) At each process region, determine the coefficients $\mathbf{c}_{k}$ of each input images by Eq. (1).

$$
\mathbf{I}_{k}=c_{k}^{1} \mathbf{I}_{1}^{B}+c_{k}^{2} \mathbf{I}_{2}^{B}+c_{k}^{3} \mathbf{I}_{3}^{B} .
$$

Then, the linear combination of $\mathbf{c}_{\mathrm{k}}$ and previously determined base images $\mathbf{I}_{1}^{\mathrm{B}}, \mathbf{I}_{2}^{\mathrm{B}}$ and $\mathbf{I}_{3}{ }_{3}$ gives linearized image $\mathbf{I}^{\mathrm{L}}$ of input image $\mathbf{I}_{\mathrm{k}}$ for each process region.

3) Select a set of linearization coefficients from the eight neighborings at the region for which no linearization coefficient set $\mathbf{c}_{\mathrm{k}}$ is found, and generate a linearized image for each process region.

4) Repeat steps 1) to 3) for each of the remaining input images.

(3) Classification of photometric factors

We can now classify photometric factors by comparing a linearized image with its corresponding input image using the classification criteria of Eq. (2).

$$
\begin{aligned}
& \text { Region }(k, p)= \\
& \begin{cases}D: & \text { if }\left(\left|i_{(k, p)}-i_{(k, p)}^{L}\right| \leq T \cdot i_{(k, p)}\right) \cap\left(i_{(k, p)} \geq T_{s}\right) \\
S: & \text { if }\left(i_{(k, p)}-i_{(k, p)}^{L}>T \cdot i_{(k, p)}\right) \\
& \bigcap\left(i_{(k, p)}^{L} \geq 0\right) \cap\left(i_{(k, p)} \geq T_{s}\right) \\
A: & \text { if }\left(i_{(k, p)}^{L}<0\right) \cap\left(i_{(k, p)}<T_{s}\right) \\
C: & \text { if }\left(i_{(k, p)}-i_{(k, p)}^{L}<-T \cdot i_{(k, p)}\right) \cap\left(i_{(k, p)}<T_{s}\right) \\
U: & \text { otherwise, }\end{cases}
\end{aligned}
$$

where, D, S, A, C, and U denote diffuse reflection, specular reflection, attached shadow, cast shadow, and undefined region respectively. The notation $\mathrm{i}_{(\mathrm{k}, \mathrm{p})}$ means the intensity of pixel $\mathrm{p}$ in the $\mathrm{k}$-th input image and $\mathrm{i}_{(\mathrm{k}, \mathrm{p})}^{\mathrm{L}}$ means the intensity of that pixel after linearization. $\mathrm{T}$ and $\mathrm{T}_{\mathrm{s}}$ denote threshold values in relation to diffuse components and shadow regions, respectively.

\section{Experimental Results}

\subsection{Effectiveness of proposed method}

We conducted a simulation to examine the effectiveness of the proposed method. At first, we used computer-generated images illuminated by a near point light source. In this experiment, four hemispheres of radius 100 $\mathrm{mm}$ with specular components were placed on a flat surface having no specular reflection. A 640x480-pixel camera with a horizontal view angle of $56^{\circ}$ was placed at $500 \mathrm{~mm}$ far from objects. The size of each process region was $80 \times 80$ pixels, which corresponds to a $5^{\circ}$ field of view.

For this experiment, we divided input images into process regions in 16 ways. Those positions were shifted 20 pixels each for $\mathrm{X}$-axis and Y-axis. In general, the optimal size of a process region depends on the distance between the light source and object, which means that process region size should be varied adaptively. However, supposing that distance between the light sources and all 


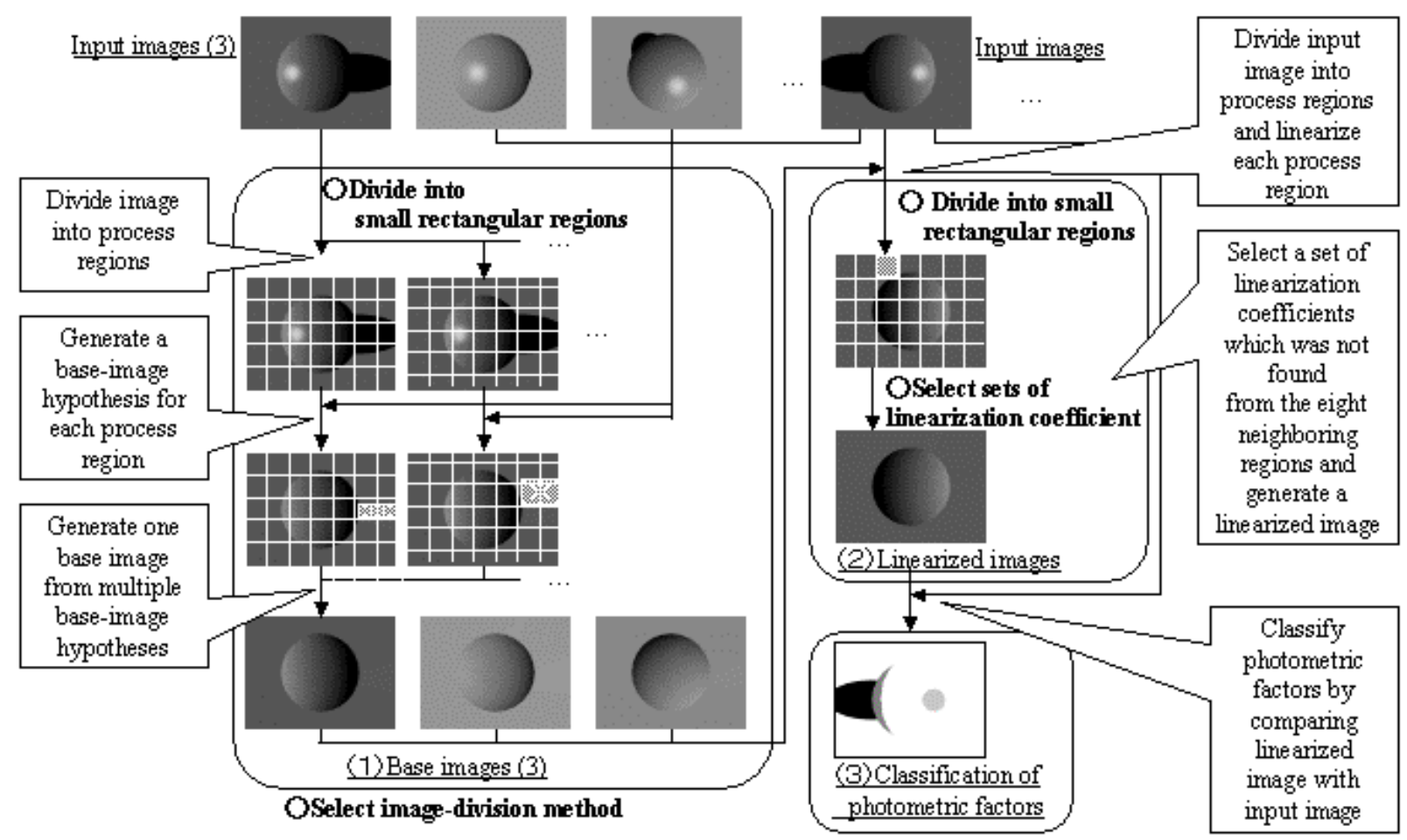

Figure 3: Process flow

points on the objects in one process region is approximately constant, a uniform process region size is acceptable. For this reason, we made no change to process region size in the experiment.

We took 24 images (examples of which are shown in Figures 4(a)-9(a)) with varying the distance between the light source and the objects from 600 to $1200 \mathrm{~mm}$. We generated linearized images and classified photometric factors by both the proposed method and the previous method [1].

In Figure 4, images (b) and (c) show the base images generated by each method. In Figures 5 and 6 , image (b) show the linearized image generated by each method; images (c)-(g) show each of the classified photometric factors; and image $(\mathrm{h})$ shows regions where classification failed. Figure 6 results that the proposed method is essentially successful in classifying specular reflection, diffuse reflection, attached shadow, and cast shadow except for boundary areas. On the other hand, the previous method classifies diffuse reflection as specular reflection over a wide area in Figures 5(c) and (d). Further, comparing result between Figures 5(h) and 6(h) denotes that the proposed method exhibits smaller error at boundary of photometric factors, but better than the previous method.

Incidentally the proposed method fails to generate a linearized image in the upper-right area of the lower-left hemisphere where shadows dominate of the input images in Figure 6(b). These results indicate that if areas dominated by shadows exist in multiple input images and if Precondition 2 and Precondition 3 are not satisfied over a wide area, the accuracy of the proposed method degrades.

Table 1 summarizes the accuracy of classifying photometric factors for all 24 input images. In this case, "classification accuracy" means percentage of successfully classified pixels out of all pixels in input images. These results show that the proposed method decrease the classification error from $20 \%$ to $6 \%$ under conditions of near point light sources.
Figure 7 shows the results of processing another type of input image (cup). These results show that the proposed method is effective even for a more complex object.

\subsection{Effectiveness of the adaptive window}

To evaluate of effectiveness of the adaptive window of process regions, we conducted the same experiment using a method that does not perform such adaptive window. This method divided input images into fixed process regions, performs processing on each of those regions, and selects the set of linearization coefficients. Figure 8 shows the result. Note that the method fails to generate linearized images in process regions dominated by specular reflection and shadows. And comparing these results with those of Figure 6 shows that the proposed method decreases the influence of this problem and generates accurate sets of linearization coefficients by generating process regions dominated by diffuse reflection by varying the position and size of process regions.

\subsection{Experiment on real image}

Figure 9 shows an example of classifying photometric factors after applying the process to real images. We used 30 real images of a diffuse-reflection sphere. These results show that the proposed method is more accurate than the previous method in classifying photometric factors even in real images.

Table 1: Experimental results

\begin{tabular}{ccc}
\hline & $\begin{array}{c}\text { Previous } \\
\text { Method [1] }\end{array}$ & $\begin{array}{c}\text { Proposed } \\
\text { Method }\end{array}$ \\
\hline $\begin{array}{c}\text { No. of successfully } \\
\text { classified pixels }\end{array}$ & 5911383 & 6965794 \\
\hline $\begin{array}{c}\text { No. of unsuccessfully } \\
\text { classified pixels }\end{array}$ & 1461417 & 407006 \\
\hline Classification accuracy & $80 \%$ & $94 \%$ \\
\hline
\end{tabular}




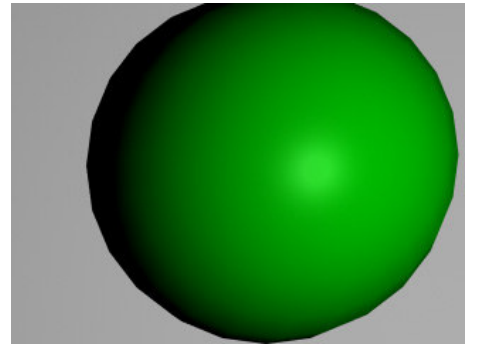

(a) Input image

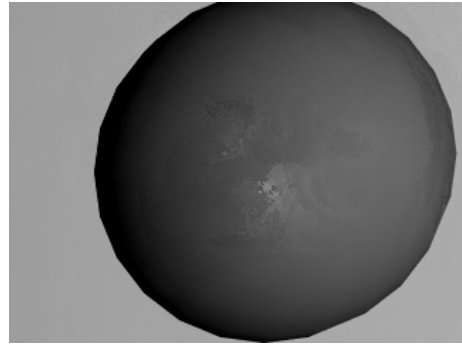

(b) Previous method Figure 4: Base images

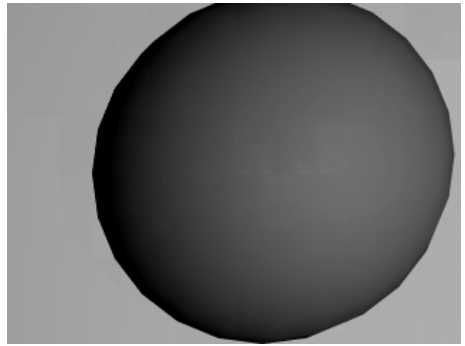

(c) Proposed method

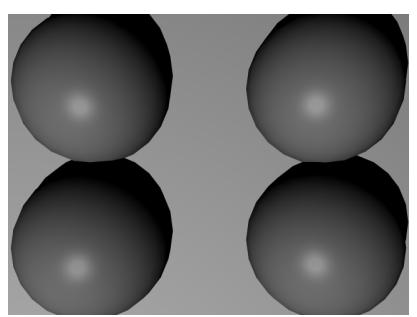

(a) Input image

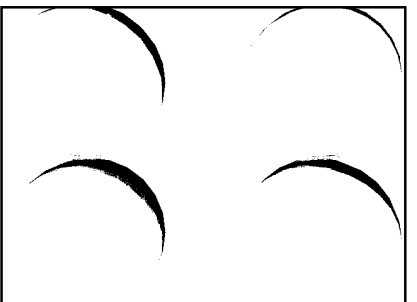

(e) Attached shadow

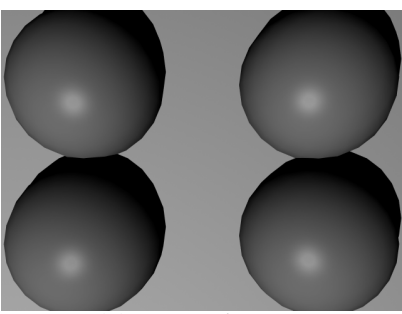

(a) Input image

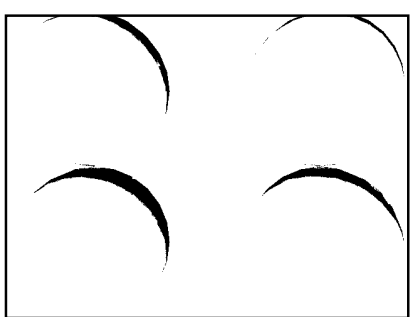

(e) Attached shadow

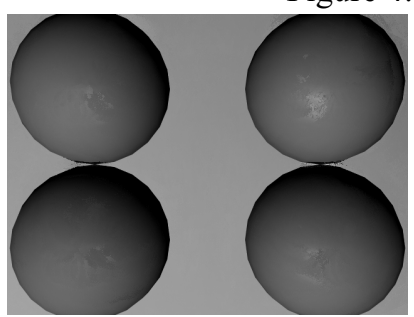

(b) Linearized image

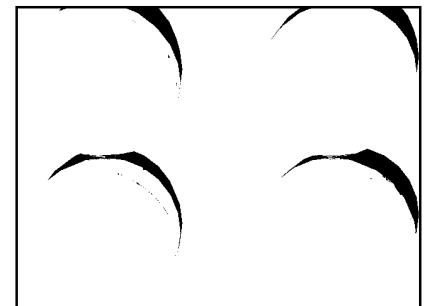

(f) Cast shadow

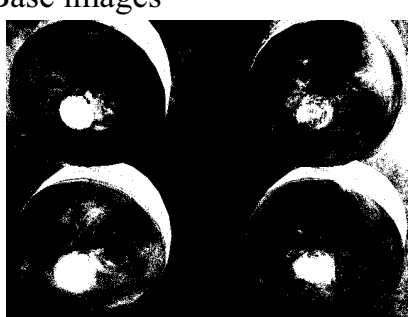

(c) Diffuse reflection

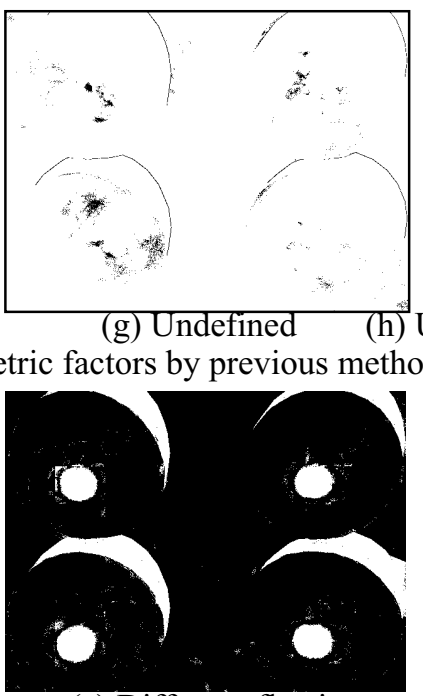

(c) Diffuse reflection

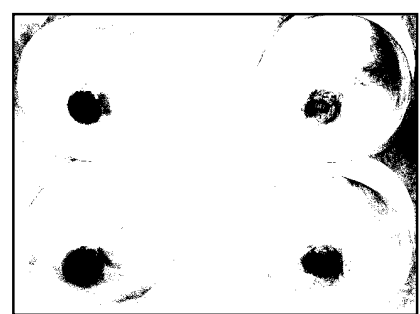

(d) Specular reflection

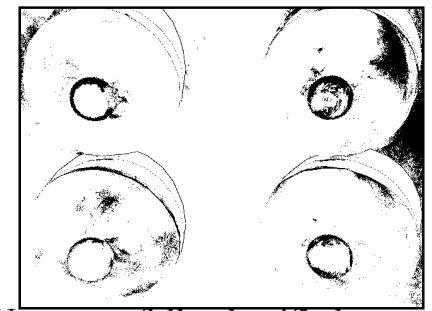

h) Unsuccessfully classified areas

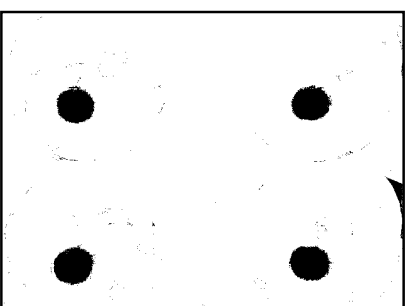

(d) Specular reflection

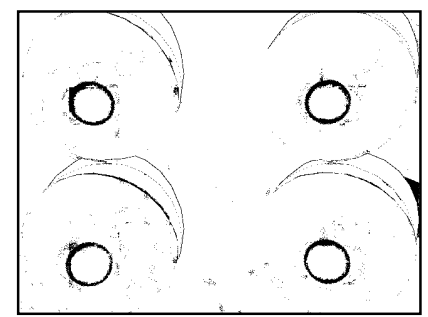

(f) Cast shadow

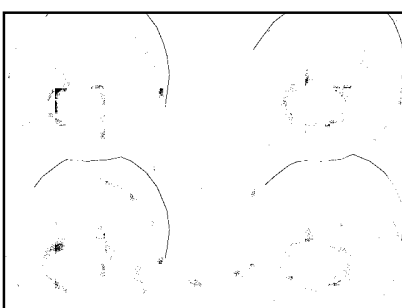

(g) Undefined

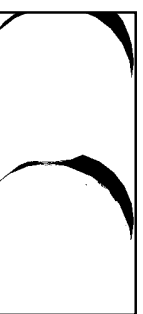

Figure 6: Classifying photometric factors by proposed method

\section{Conclusion}

In this paper, we proposed a photometric linearization method that can be used even under near point light sources. This method divides images into small regions and selects the sets of linearization coefficients from those regions. The position and size of process regions varies, and then combining optimal regions generates base images. Using computer-generated images, we quantitatively showed that the proposed method can generate accurate linearized images and decrease classification error under near point light sources. Further, we showed that the proposed method is effective for real images.

\section{References}

[1] M. A. Fischler and R. C. Bolles: "Random Sample Consensus: A Paradigm for Model Fitting with Applications to Image Analysis and Automated Cartography", Communications of the ACM, Vol. 24, Issue 6, pp. 381-395, 1981.

[2] K. Ikeuchi, and K. Sato: "Determining Reflectance Properties of an Object Using Range and Brightness Images", IEEE Transactions on Pattern Analysis and Machine Intelligence, 


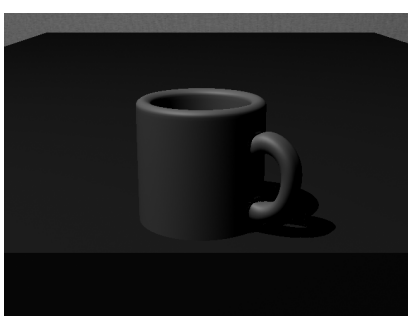

(a) Input image

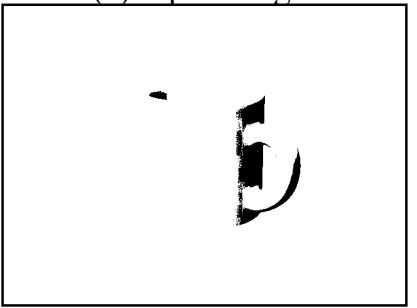

(e) Attached shadow

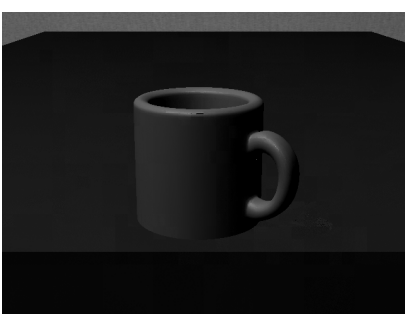

(b) Linearized image

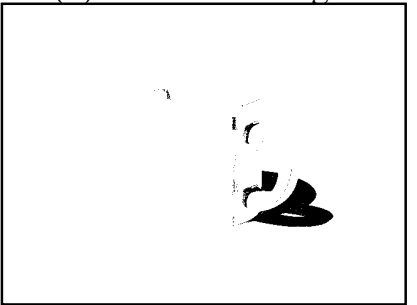

(f) Cast shadow

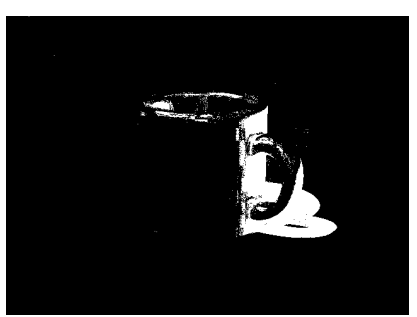

(c) Diffuse reflection

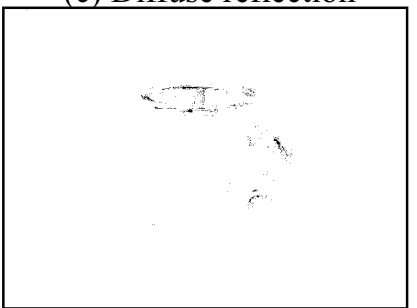

(g) Undefined

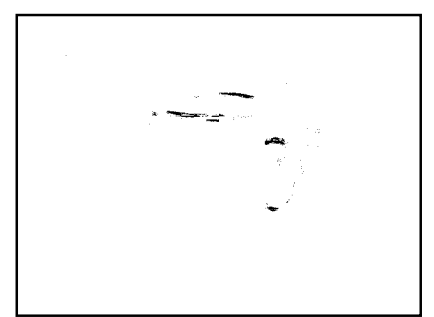

(d) Specular reflection

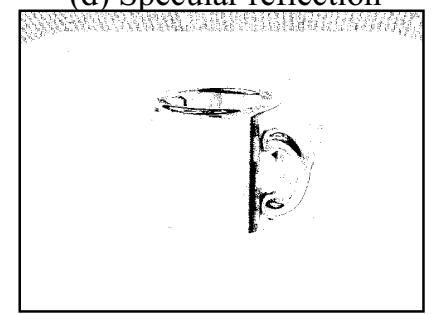

(h) Unsuccessfully classified areas

Figure 7: Classifying photometric factors by proposed method (cup)

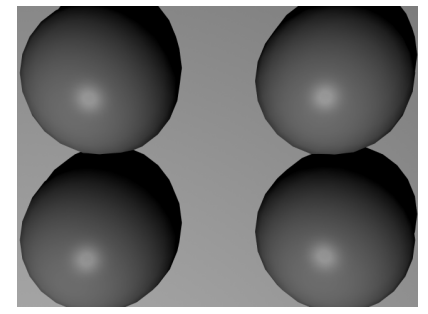

(a) Input image

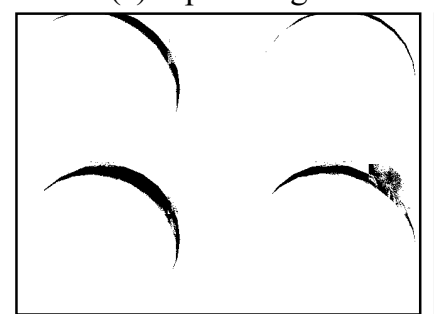

(e) Attached shadow

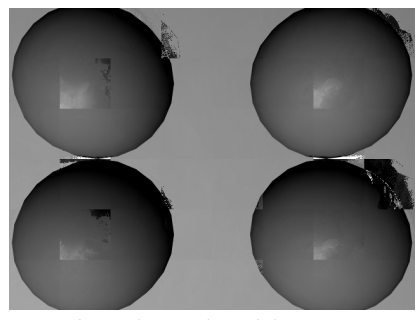

(b) Linearized image

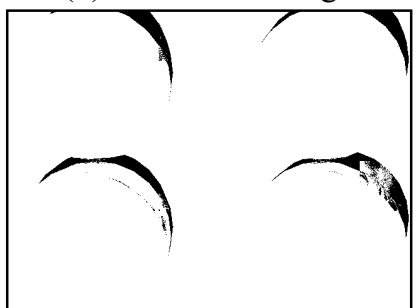

(f) Cast shadow

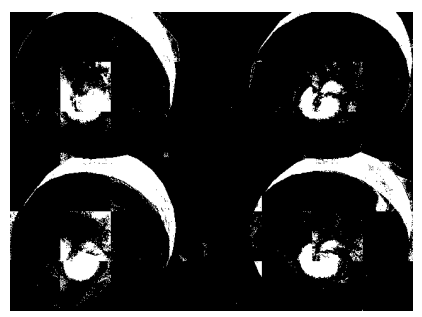

(c) Diffuse reflection

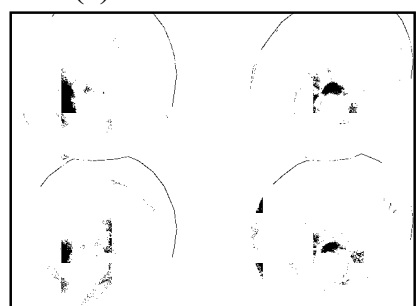

(g) Undefined

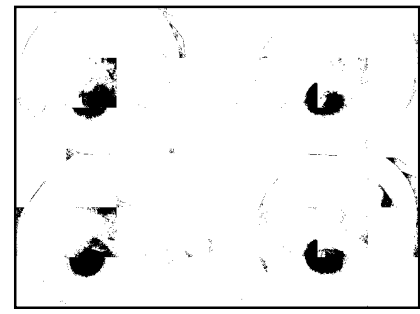

(d) Specular reflection

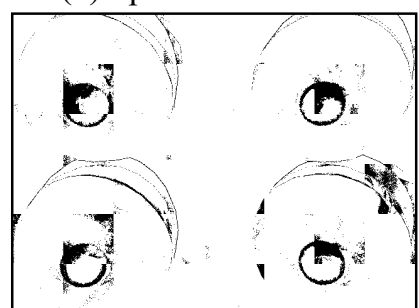

(h) Unsuccessfully classified areas

Figure 8: Classifying photometric factors by fixed process regions method

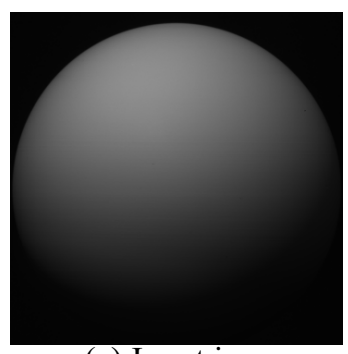

(a) Input image

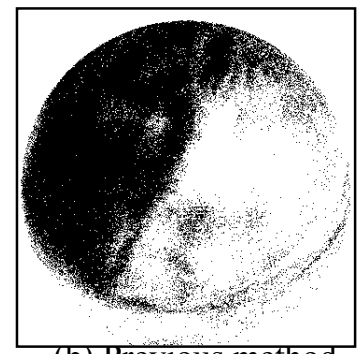

(b) Previous method

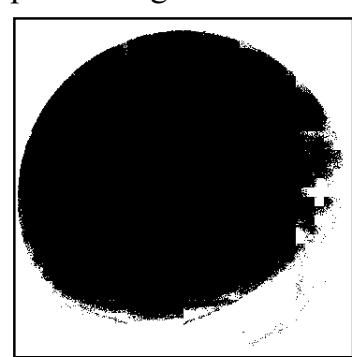

(c) Proposed method

Figure 9: Results of classifying a diffuse reflection region

vol.13, no.11, pp.1139-1153, 1991

[3] Y. Ishii, K. Fukui, Y. Mukaigawa and T. Shakunaga, "Photometric Linearization Based on Classification of Photometric Factors," IPSJ Trans. on CVIM, Vol. 44, No. SIG5 (CVIM6), pp. 11-21, 2003. (In Japanese)

[4] Y. Mukaigawa, H. Miyaki, S. Mihashi and T. Shakunaga: "Photometric Image-Based Rendering for Realizing Virtual Lighting Conditions in Image Synthesis," IPSJ Trans. on CVIM, Vol. 41, No. SIG10 (CVIM1), pp. 19-30, 2000. (In Japanese))

[5] Shashua,A: "Geometry and Photometry in 3D Visual Recognition," Ph.D. thesis, Dept. Brain and Cognitive Sceience, NIT, 1992.
[6] R. Swaminathan, S. B. Kang, R. Szeliski, A. Criminisi, and S. K. Nayar: "On the Motion and Appearance of Specularities in Image Sequences", Proc. Of ECCV, vol.1, pp.508-523, 2002

[7] S. Umeyama and G. Godin: "Separation of Diffuse and Specular Components of Surface Reflection by Use of Polarization and Statistical Analysis of Images", IEEE Transactions on Pattern Analysis and Machine Intelligence, vol.26, no.5, pp.639-647, 2004

[8] O. Yamaguchi and K. Fukui: "Appearance Matching by Qualitative Trinary Representation," IEICE Technical Report, PRMU, Vol. 2002-34, pp. 23-30, 2002. (In Japanese) 